$\xi=-1$

\title{
Fuzzy logic-based improved ventilation system for the pharmaceutical industry
}

\author{
Sam Matiur Rahman ${ }^{1}$, Mohammad Fazle Rabbi ${ }^{2}$, Omar Altwijri ${ }^{3}$, Mahdi Alqahtani ${ }^{3}$, Tasriva Sikandar ${ }^{2}$, Izzeldin \\ Ibrahim Abdelaziz ${ }^{2}$, Md. Asraf Ali ${ }^{1}$ *, Kenneth Sundaraj ${ }^{4}$ \\ ${ }^{1}$ Department of Software Engineering, Daffodil International University, Dhaka, Bangladesh \\ ${ }^{2}$ Faculty of EEE, Universiti Malaysia Pahang, 26600-Pekan, Pahang, Malaysia \\ ${ }^{3}$ Biomedical Technology Department, College of Applied Medical Sciences, King Saud University, Riyadh, Saudi Arabia \\ ${ }^{4}$ Faculty of Electronics and Computer Engineering, Universiti Teknikal Malaysia Melaka, Durian Tunggal, Melaka, Malaysia \\ *Corresponding author E-mail: asrafbabu@hotmail.com
}

\begin{abstract}
Indoor air quality in pharmaceutical industry plays a vital role in the production and storing of medicine. Stable indoor environment including favorable temperature, humidity, air flow and number of microorganisms requires consistent monitoring. This paper aimed to develop a fuzzy logic-based intelligent ventilation system to control the indoor air quality in pharmaceutical sites. Specifically, in the proposed fuzzy inference system, the ventilation system can control the air flow and quality in accordance with the indoor temperature, humidity, air flow and microorganisms in the air. The MATLAB® fuzzy logic toolbox was used to simulate the performance of the fuzzy inference system. The results show that the efficiency of the system can be improved by manipulating the input-output parameters according to the user's demands. Compared with conventional heating, ventilation and air-conditioning (HVAC) systems, the proposed ventilation system has the additional feature of the existence of microorganisms, which is a crucial criterion of indoor air quality in pharmaceutical laboratories.
\end{abstract}

Keywords: Fuzzy Inference System; Air-Conditioning; Pharmaceutical Laboratories; Smart Ventilation.

\section{Introduction}

The ventilation system plays an important role in the pharmaceutical industry. Without a ventilation system, the quality of medicines may degrade due to fluctuations in the number of microorganisms and an increase in the temperature of the indoor environment. The purpose of a ventilation system in the pharmaceutical industry is to provide a sufficient flow of clean air. The system should be able to fulfil the temperature and humidity requirements of a clean room [1]. The air quality depends on the surrounding temperature, air humidity, dust conditions, and microorganism, among other factors. A previous study [2] investigated four pharmaceutical laboratories in Malaysia and compared their air qualities with the American Society of Refrigerating Engineers (ANSI/ASHRAE) standard [3], and the findings revealed that at least two laboratories could not maintain the recommended air quality.

However, the room temperature and air humidity cannot be easily modelled mathematically. Therefore, the complicated effects of input variables, such as temperature, air humidity, and microorganisms, can be predicted by artificial intelligence (AI) to make an immediate decision regarding the output variables, such as fan speed, operation mode and air conditioning, based on some setting rules. For example, two previous studies [4], [5] proposed fuzzy logic-based improved ventilation systems for patient monitoring during surgical operation, and since then, fuzzy logic-based systems have been used in various medical applications. A previous investigation [5] showed that the use of a fuzzy logic-based ventilation system during general anaesthe- sia is safe for patients with different ages and diseases and undergoing diverse surgical procedures. Additionally, a previous study [4] investigated the use of fuzzy logic-based patient monitoring and alarming systems by anaesthesiologists during highly invasive surgical procedures. In this sequel, [6], [7] also investigated fuzzy logic-based controller design for the weaning process for ventilated patients.

A proper heating, ventilation and air conditioning (HVAC) system is a critical part of pharmaceutical cleanroom design [8]. Although various design guidelines and standards are available, there are no clear-cut guidelines for many crucial HVAC design parameters, particularly air changes per hour for a specific class of cleanrooms. The Food and Drug Administration (FDA) guidelines only specify a minimum of twenty air changes per hour for controlled areas but do not provide any specifics. A published article [9] discusses a method for the optimal selection and management of HVAC systems in new and existing buildings. The method utilizes a combination of two analysis tools, multi-criteria decision-making and building simulation, for a holistic assessment of HVAC systems. The method of entransy analysis for optimizing heat-transfer processes was previously used [10] for cooling and heating, and it has been shown that the entransy dissipation principle and the minimum thermal resistance principle are suitable for analysing the heat transfer process in an HVAC system.

Authors in [11] discussed several technologies for genetic analysis that have been used to rapidly detect microbial contamination, to provide a more accurate identification of microorganisms, to understand the microbial diversity in cleanroom environments and to optimize pharmaceutical processes. The sus- 
tainability conditions of pharmaceutical companies were previously investigated considering the annual reports of eleven pharmaceutical companies [12]. Some researchers [13] investigated the use of a multi-criteria analysis for the design of a HVAC system. When designing the system's economics, energy and environment, the user's satisfaction was considered. An auto-tuning receding-horizon optimization method was previously proposed [14] to synthesize a proportional-integralderivative (PID)-type controller for air-handling units (AHUs). This algorithm comprises two levels of control: the lower level uses a conventional PID controller to obtain an acceptable, but not necessarily optimal, performance, and the higher level provides optimal low-level controller parameters through minimization of the generalized predictive control criterion.

A fuzzy logic-based system has been developed for the maintenance of the appropriate indoor air conditions [15]. In the study, the basic parameters of air conditioning and the oxygen level were considered as inputs for the fuzzy inference system. In addition, [16], [21], [22] investigated fuzzy logic controller design for other intelligent manufacturing technology. Different types of contamination and health hazards in pharmaceutical industries have been investigated in previous studies [17-20] The microbial risk to aseptically manufactured products in pharmaceutical cleanrooms can be assessed using fundamental equations that model the dispersion, transfer and deposition of microbial contamination [17]. This assessment can be performed in two stages: the first stage assesses the transfer of contamination from all the sources within the cleanroom suite, and the second stage assesses both air and surface contact contamination within critical production areas. A previous study [18] investigated a cleanroom contamination control system and showed that both the route underlying the transfer of contaminants and the source of contamination should be properly addressed in pharmaceutical cleanrooms. In addition, the assessment of microbial risk in pharmaceutical cleanrooms has been previously investigated [19].

In this study, a fuzzy logic controller was used with a set of range and membership functions for each input and output variable. The set of rules was fixed after observing the changes in the surface viewer. The effect of changes in the input variables on the output variables was elaborately discussed. The MATLAB ${ }^{\circledR}$ fuzzy logic toolbox was used to simulate the inference system

\section{Study design}

\subsection{System configuration}

Figure 1 shows a simple block diagram of a fuzzy logic controller. Four input variables with specific membership functions are required for fuzzification, and these include temperature, humidity, microorganisms and air movement. If-then rules are used to define the fuzzy rules and thereby set a rule base for decisionmaking logic. In fuzzification, the centroid method is used to obtain various outputs, including the fan speed, air conditioning and mode.

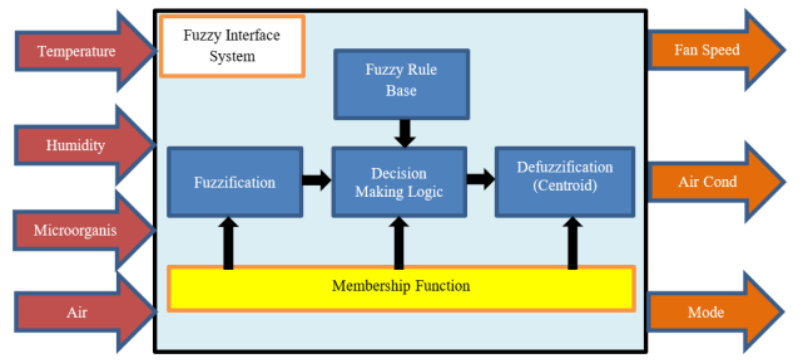

Fig. 1: Block Diagram of the Fuzzy Logic Controller.
The first step in the fuzzy inference process is fuzzification, which consists of the transformation of crisp inputs into membership values regarding the linguistic terms in all fuzzy sets. The membership values obtained from the transformation from crisp inputs are called fuzzy inputs and are needed for the next step of the fuzzy inference process. A set of fuzzy rules for decision making are built in the second step. A decision is then made for a given input, and if-then rules explain why a decision is made for a given input. The if-then rules are given data during the IF part of process and are executed during the THEN part of the process. Defuzzification is the last step of the fuzzy inference process and consists of the transformation of fuzzy outputs, which are membership values regarding the linguistic terms, into crisp outputs. Numerous methods for defuzzification, including centroid, bisector, and mean maximum value, have been developed. The defuzzification method used in this study is the centroid method, which is used to define the centroids of sets. Figure 2 shows the settings of the fuzzy inference system.

\begin{tabular}{|c|c|c|c|c|}
\hline FIS Name: & Ventilation-System & & FIS Type: & mamdani \\
\hline \multirow{5}{*}{$\begin{array}{l}\text { And method } \\
\text { Or method } \\
\text { Implication } \\
\text { Aggregation } \\
\text { Defuzzification }\end{array}$} & $\min$ & $\checkmark$ & \multirow{4}{*}{$\begin{array}{l}\text { Current Variable } \\
\text { Name } \\
\text { Type } \\
\text { Range }\end{array}$} & \\
\hline & $\max$ & 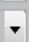 & & \\
\hline & $\min$ & $\checkmark$ & & \\
\hline & $\max$ & $\checkmark$ & & \\
\hline & centroid & $\checkmark$ & Help & Close \\
\hline
\end{tabular}

Fig. 2: Settings of the Fuzzy Inference System.

\subsection{System parameters and fuzzy rules}

The parameters of the system, including the linguistic variables and the input and output terms, are defined in Tables 1 and 2, respectively. The principal step of the fuzzification process is the definition of the fuzzy sets in the input and output variables. The possible domain interval of both the inputs and outputs are divided into several regions such that they overlap or do not overlap with each other. The length of the region may differ for each variable, and one membership function is assigned to each region. To control the system, we divided the input 'temperature' into cold, warm and hot values based on the indoor environment and are within the range of -10 to $+10{ }^{\circ} \mathrm{C}$. Any value above this range will be assumed to equal infinity. In a similar manner, we divided the input 'humidity into dry, moderate and humid values in the range of 5 to $85 \%$.

The parameter 'microorganism' was divided into 'low', 'medium' and 'high' in the range of 0 to 5 colony-forming units (cfus) $/ \mathrm{m}^{3}$. Additionally, the input parameter 'air movement' was set as 'slow', 'medium' and 'fast' in the range of 0 to $10 \mathrm{~m}^{3} / \mathrm{min}$, and the output variables 'fan speed' and 'cold air' were divided according to the speed range required into five different categories, namely 'very slow', 'slow', 'medium', 'fast' and 'very fast' The minimum and maximum fan speeds were set to 0 and 1800 rpm (revolutions per minute), and the corresponding values for cold air were 0 and $18 \mathrm{~m}^{3} / \mathrm{min}$, respectively. In addition, another output parameter, the 'operation mode', has three different statuses, specifically 'dehumidifier', 'normal' and 'purification'. In general, there is no standard method for selecting the proper shape of the fuzzy sets of the control variables [18], although trial-and-error methods are usually exercised.

Table 1: Input Parameters

\begin{tabular}{lll}
\hline Parameter & Statuses & Units \\
\hline Temperature & Cold, Warm, Hot & -10 to $+10{ }^{\circ} \mathrm{C}$ \\
Humidity & Dry, Moderate, Humid & 5 to $85 \%$ \\
Microorganism & Low, Medium, High & 0 to $5 \mathrm{cfu} / \mathrm{m}^{3}$ \\
Air movement & Slow, Medium, Fast & 0 to $10 \mathrm{~m}^{3} / \mathrm{min}$ \\
\hline
\end{tabular}


Table 2: Output Parameters

\begin{tabular}{|c|c|c|}
\hline Parameter & Statuses & Units \\
\hline Fan speed & $\begin{array}{l}\text { Very slow, slow, medium, fast, very } \\
\text { fast }\end{array}$ & $\begin{array}{l}0 \text { to } 18,00 \\
\text { rpm }\end{array}$ \\
\hline Cold air & $\begin{array}{l}\text { Very slow, slow, medium, fast, very } \\
\text { fast }\end{array}$ & $\begin{array}{l}0 \text { to } 18 \\
\mathrm{~m}^{3} / \mathrm{min}\end{array}$ \\
\hline $\begin{array}{l}\text { Operation } \\
\text { mode }\end{array}$ & Dehumidifier, Normal, Purification & 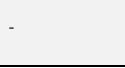 \\
\hline
\end{tabular}

As shown in Figure 3(a), the input variable "TempDiff" was given three membership functions: cold, [-18 -10 -2]; warm, [-8 0 8]; and hot, [2 10 18]. Figure 3(b) presents the input variable "Humidity" with the following three membership functions: dry, [14 45]; moderate, [14 65]; and humid, [14 85.2]. Figure 3(c) presents the input variable "Microorganism", which has three membership functions: low, [-2 -0.2 0.2 2]; medium, [0.763 2.51 4.26]; and high, [ [ $\left.\begin{array}{llll}3 & 4.8 & 5.2 & 7\end{array}\right]$. Figure 3(d) presents the input variable "AirMovement" with the following membership functions: slow, [0.4 3.6]; medium, [0.5 46 9.5]; and fast, [6.426 9.626]

In addition, Figure 3(e) shows the output variable "AirCondition", which has five membership functions: very slow, [-4 -0.45 1.5 7]; slow, [2 7 8.5]; medium, [6 9 12]; fast, [9.5 11 16]; and

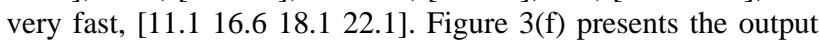
variable "FanSpeed" with five membership functions: very slow, [-450 0 450]; slow, [0 450 900]; medium, [450 900 1350]; fast, [900 1350 1800]; and very fast, [1350 1800 2250]. Figure 3(g) presents the output variable "Mode" with three membership functions: dehumidifier, [-10 -5 15 40]; normal, [5 4060 95]; and purification, [60 85105110$]$
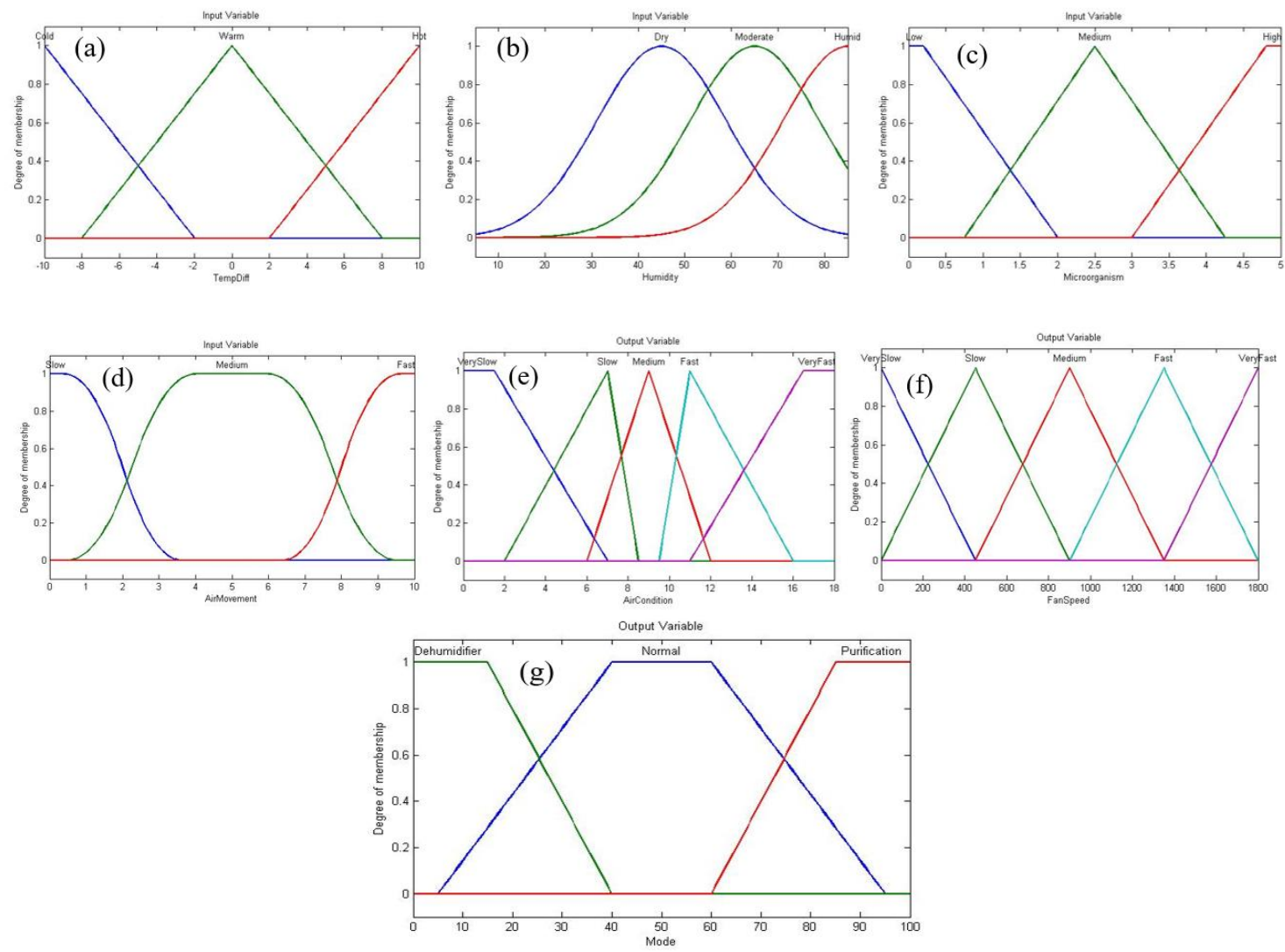

Fig. 3: Input variables: (A) “Tempdiff”, (B) "Humidity”, (C) "Microorganism”, (D) and “Airmovement”. Output Variables: (E) “Aircondition”, (F) "Fanspeed" and (G) Mode.

\section{Results}

Figure 4 shows the rule viewer for the fuzzy logic-based ventilation system, which is used to identify the crisp output for a given input through the fuzzy inference process. Samples of input variables were used to identify the ventilation system. In general, the subtropical zone of Earth has an average temperature of $25^{\circ} \mathrm{C}$ and a humidity of $60 \%$. Table 3 shows the results of changes in humidity. The actual values of Temperature, Microorganism and AirMovement were fixed to 27.5, 2.5 and 5, respectively. The optimal temperature is $25^{\circ} \mathrm{C}$, and as a result, TempDiff equals $2.5^{\circ} \mathrm{C}$. As shown in the table, the humidity has a lower effect on controlling the air conditioning system and fan speed but a marked effect on the mode of the system. As the humidity increased from 'dry' to 'humid', the mode shifted toward the 'dehumidifier' mode from the 'normal' mode.
Figure 6(a) shows the effect of the temperature difference and the humidity on air conditioning. The results show that an increase in the temperature difference will increase the air conditioning flow rate. In addition, the humidity only yields temperature-induced effects on the air conditioning at values lower than 40\%: an increase in the temperature difference causes an unsteady increase in the air conditioning flow rate.

Figure 6(b) shows the effect of the temperature difference and microorganisms on air conditioning. Specifically, an increase in the temperature difference increases the air conditioning flow rate, and microorganisms increase the air conditioning flow rate when the temperature differences are not in the ranges of [-1 1] and $\left[\begin{array}{ll}5 & 10]\end{array}\right]$. At the range [ -11 ], a constant air conditioning flow rate was obtained, and at [5 10], an increase in microorganisms decreases the air conditioning.

Figure 6(c) shows the effect of the temperature difference and the humidity on the fan speed. The graph shows that the relationship between the humidity and the temperature difference on the fan speed is very similar to that between the humidity and 
temperature difference on air conditioning. Figure 6(d) shows the effect of microorganisms and humidity on fan speed, and Figure 6(e) illustrates the effect of microorganisms and humidity on mode. As shown in the graph, the relationship between microorganisms and humidity on mode was inconsistent. At humidity values less than $60 \%$ and microorganism values less than 3 , a constant mode value of 50 was obtained. In contrast, at a microorganism value in the range of [3 to 4], the mode exhibited a decrease, and at a humidity level higher than $60 \%$, the value of mode presented a marked decrease except when the microorganism value was greater than 3.5.

Table 4 shows the results of changes in the actual temperature. The humidity was $60 \%$, the value of the variable Microorganism was 2.5, and the value for the variable AirMovement equalled 5 . The optimal temperature was $25^{\circ} \mathrm{C}$; as a result, the TempDiff depended on the actual temperature. As demonstrated in the table, the difference in temperature has a lower effect on controlling the mode of the system but a marked effect on the air conditioning and fan speed. If the temperature difference corresponded to the 'warm' membership function, the air conditioning and fan speed were assigned to the 'medium' membership function, and as the temperature difference increases from 'warm' to 'hot', the air conditioning and the fan speed increase to 'very fast'.

\section{Discussion}

The use of a ventilation system in the pharmaceutical industry is required for maintaining the environment as close as possible to the ideal environment for the production and storage of the target medicine. Fuzzy logic is one of the methods that can control the ventilation machine based on the temperature difference between actual and ideal, the humidity, the existing microorganisms and air movement. The ventilation system then controls the air conditioning, fan speed and ventilation mode to maintain the environment at a suitable condition. The surface viewer is used to display the relationship of an output variable with one or two input variables through 2D and 3D surface plots.

Figure 5 shows the relationship between an input variable and an output variable. In particular, Figure 5(a) shows the effect of the temperature difference on air conditioning and demonstrates that an increase in the temperature difference will increase the air conditioning flow rate and that various ranges of temperature

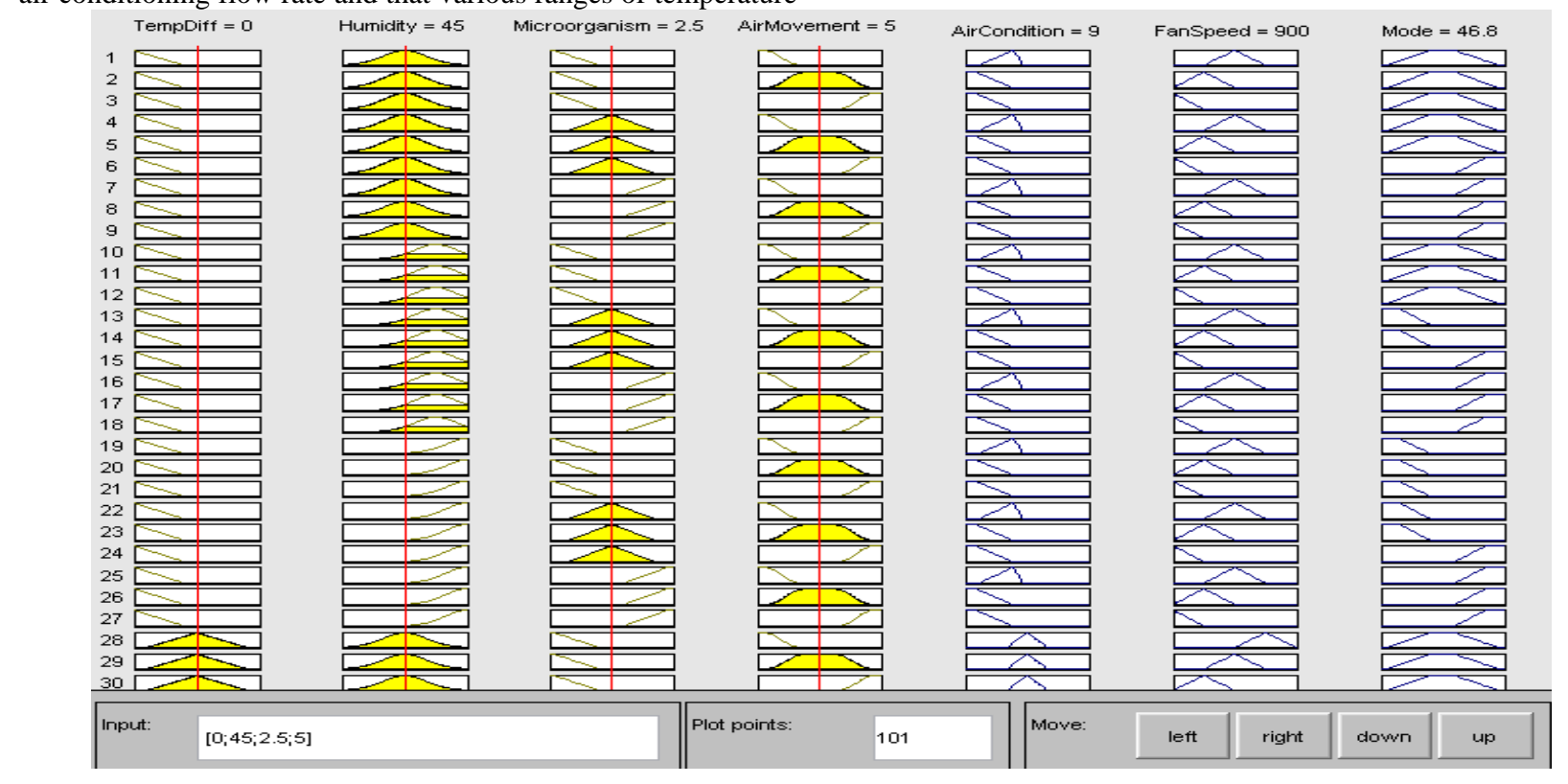

Fig. 4: Rule Viewer of the Developed Fuzzy Inference System (FIS). differences, specifically [ -10 to -8.5$],[-1.5$ to 1.5$]$ and [8.5 to $10]$, result in a constant air conditioning flow rate. Figure 5(b) shows the effect of the temperature difference on the fan speed, and the relationship between the fan speed and temperature difference is very similar to that between the air conditioning flow rate and the temperature difference. Figure 5(c) illustrates the effect of air movement on fan speed, demonstrating that an increase in the air movement will decrease the fan speed, although the rate at which the speed is reduces is not constant. The effect of the temperature difference on mode is shown in Figure 5(d), which illustrates that an increase in the temperature difference will yield first an increase and then a decrease in the modes. As shown in Figure 5(e), the analysis of the effect of humidity on mode reveals that an increase in the humidity will decrease the value of mode. At a humidity higher than $80 \%$, the mode value decreases linearly. Figure 5(f) shows the effect of microorganisms on mode, demonstrating that an increase in microorganisms to values from 3.5 to 4.25 will markedly increase the mode value and further increment to the value higher than 4.25 will not change the mode much.

\section{Conclusion}

A fuzzy logic controller with a fuzzy inference system was developed for air flow control in the pharmaceutical industry, specifically to ensure maintenance of the air quality during production. The purpose of the ventilation system proposed in this study was to control the air conditioning flow rate, fan speed and system mode and thereby obtain suitable values for the environment temperature, humidity, microorganisms and air flow rate in the pharmaceutical industry. Future studies should use hybrid intelligent systems, such as a neuro-fuzzy system. In the proposed system, the membership function of each input was generated by a MATLAB $®$-based adaptive neuro-fuzzy inference system with given sets of data for the ideal conditions. In this process, the number of membership functions and the type of membership functions can be selected according to the user's demands. Additionally, the rules can be manipulated according to the membership functions and the input variables, yielding a more convenient and efficient system in terms of time consumption and user adaptability for the pharmaceutical industry. 
Table 3: Results of the Rule Viewer (Humidity)

\begin{tabular}{|c|c|c|c|c|}
\hline $\begin{array}{c}\text { TempDiff } \\
\left({ }^{\circ} \mathbf{C}\right)\end{array}$ & $\begin{array}{c}\text { Humidity } \\
(\mathbf{\%})\end{array}$ & $\begin{array}{c}\text { AirCondition } \\
\left(\mathbf{m}^{\mathbf{3}} \mathbf{\text { min }}\right)\end{array}$ & $\begin{array}{c}\text { FanSpeed } \\
(\mathbf{r p m})\end{array}$ & Mode \\
\hline 2.5 & 15 & 11.4 & 1060 & 49.9 \\
\hline 2.5 & 40 & 9.75 & 943 & 48.2 \\
\hline 2.5 & 60 & 9.75 & 943 & 40.7 \\
\hline 2.5 & 80 & 9.75 & 943 & 20.9 \\
\hline 2.5 & 85 & 9.75 & 943 & 17.9 \\
\hline
\end{tabular}

Table 4: Results of the Rule Viewer (Tempdiff)

\begin{tabular}{|c|c|c|c|c|}
\hline $\begin{array}{c}\text { Temperature } \\
\left({ }^{\circ} \mathbf{C}\right)\end{array}$ & $\begin{array}{c}\text { TempDiff } \\
\left({ }^{\circ} \mathbf{C}\right)\end{array}$ & $\begin{array}{c}\text { AirCondition } \\
\left(\mathbf{m}^{\mathbf{3}} / \mathbf{m i n}\right)\end{array}$ & $\begin{array}{c}\text { FanSpeed } \\
(\mathbf{r p m})\end{array}$ & Mode \\
\hline 23 & -2 & 9 & 900 & 39.9 \\
\hline 27 & 2 & 9 & 900 & 39.9 \\
\hline 29 & 4 & 11.4 & 1050 & 43 \\
\hline 32 & 7 & 14.3 & 1270 & 41.6 \\
\hline 34 & 9 & 15.5 & 1350 & 38.5 \\
\hline
\end{tabular}

(A)

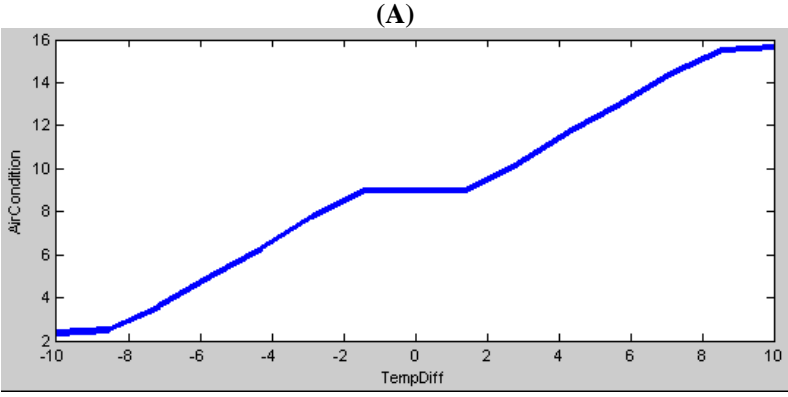

(B)

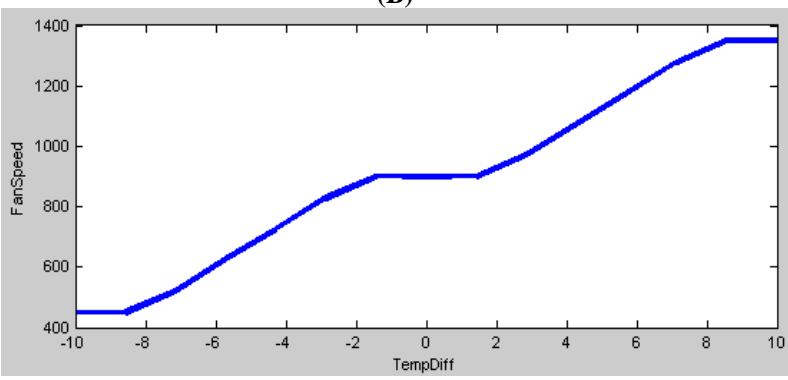

(C)

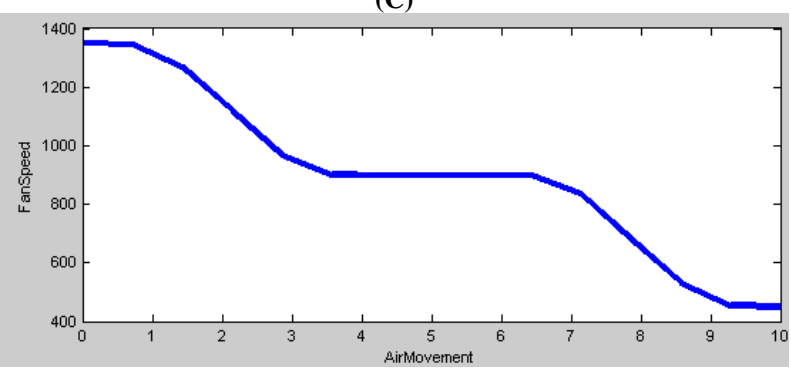

(D)

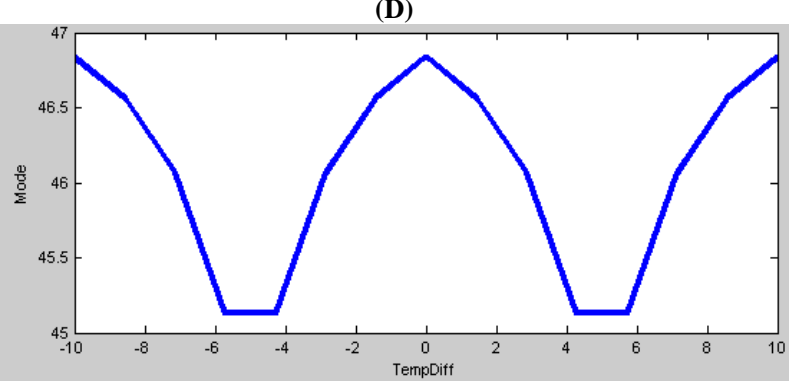

(E)

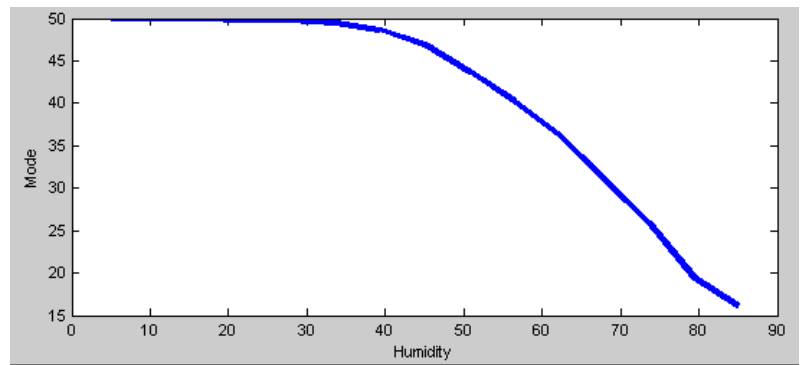

(F)

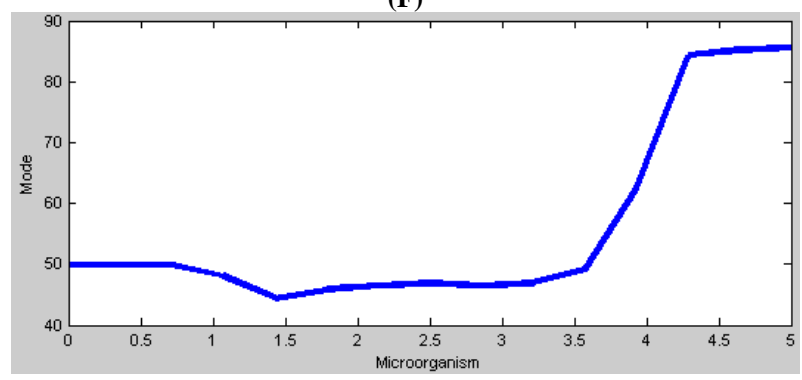

Fig. 5: 2D Surface Viewers for (A) Air Conditioning vs. Temperature Difference, (B) Fan Speed vs. Temperature Difference, (C) Fan Speed vs. Air Movement, (D) Mode vs. Temperature Difference, (E) Mode vs. Humidity, and (F) Mode vs. Microorganisms.

(A)

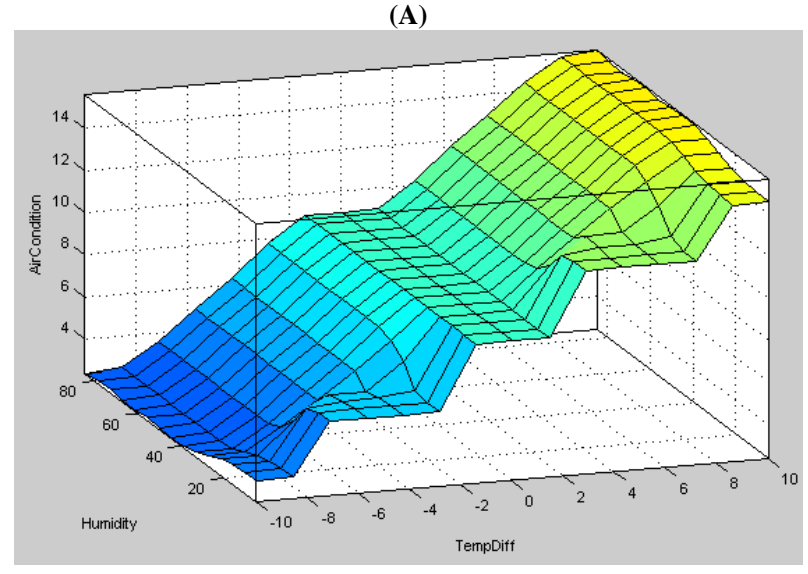

(B)

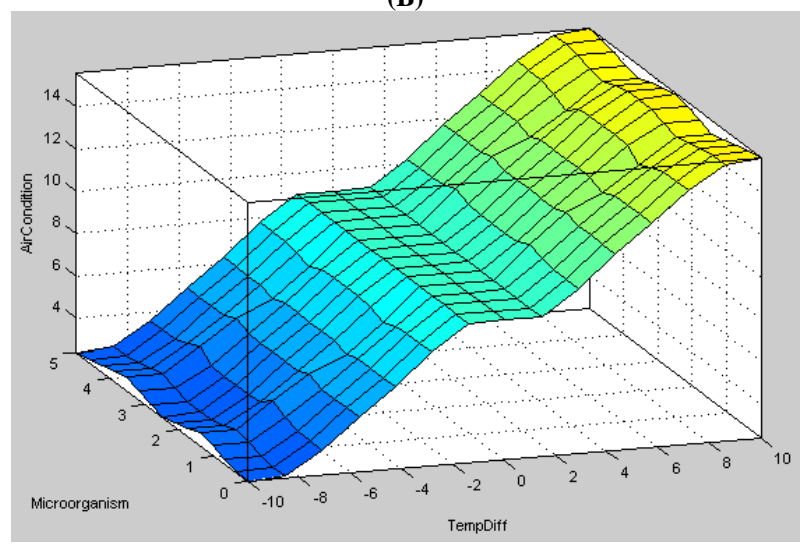

(C) 


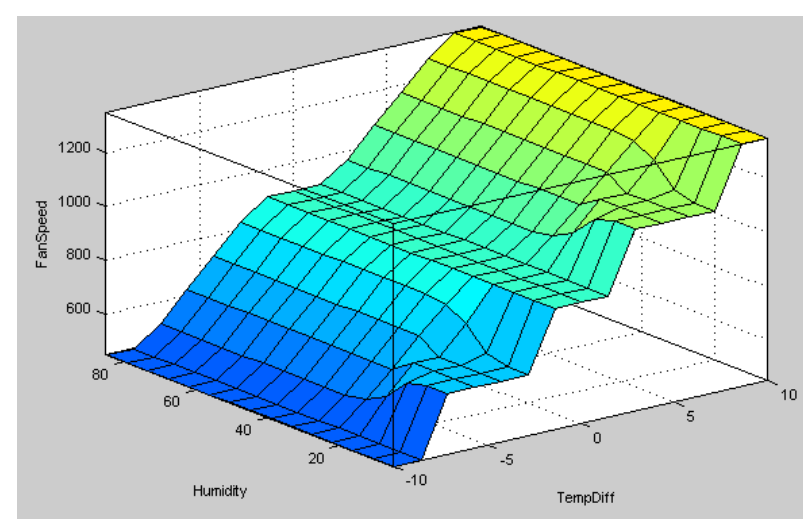

(D)

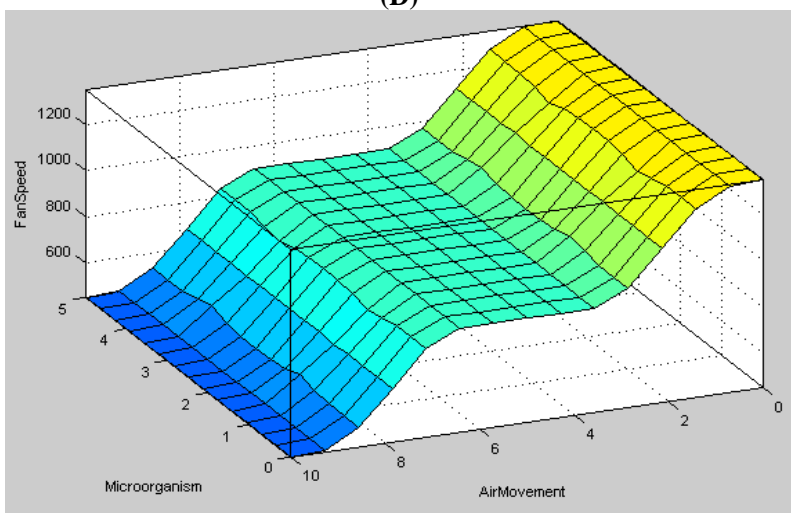

(E)

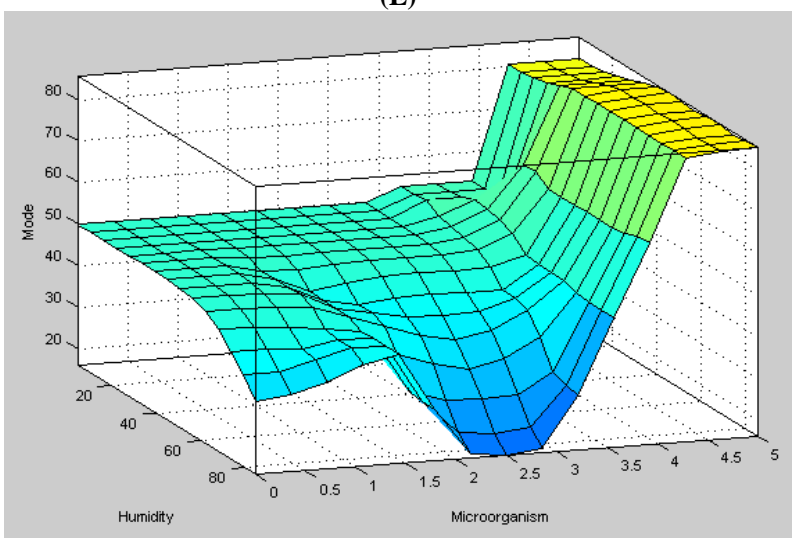

Fig. 6: 3D Surface Viewer for (A) the Effect of Humidity and Temperature Difference on Air Conditioning, (B) the Effect of Microorganisms and Temperature Difference on Air Conditioning, (C) the Effect of Humidity and Temperature Difference on Fan Speed, (D) the Effect of Microorganisms and Air Movement on Fan Speed, And (E) the Effect of Humidity and Microorganisms on Mode.

\section{Acknowledgement}

The authors extend their appreciation to the College of Applied Medical Sciences Research Center and the Deanship of Scientific Research at King Saud University for funding this research.

\section{References}

[1] Schneider RK (2001), Designing Clean Room HVAC Systems ASHRAE journal. 43(8):39.

[2] Yau YH, Chew BT, Saifullah AZ (2012), Studies on the indoor air quality of Pharmaceutical Laboratories in Malaysia. International Journal of Sustainable Built Environment. 1(1):110-24. https://doi.org/10.1016/j.ijsbe.2012.07.005.

[3] Clement JD (1996). Reproductive health hazards in the pharmaceutical industry. Occupational medicine (Philadelphia, Pa.).12(1):13143.
[4] ANSI/ASHRAE Standard 55, (2004). Thermal Environmental Conditions for Human Occupancy. American Society of Heating, Refrigerating and Air-Conditioning Engineers, Atlanta GA.

[5] Becker K, Thull B, Käsmacher-Leidinger H, Stemmer J, Rau G, Kalff G, Zimmermann HJ (1997), Design and validation of an intelligent patient monitoring and alarm system based on a fuzzy logic process model. Artificial intelligence in medicine. 11(1):33-53. https://doi.org/10.1016/S0933-3657(97)00020-1.

[6] Kilic U, Gulluoglu MT, Guler H, Kaya T (2016), Analysis of Pulmonary and Hemodynamic Parameters for the Weaning Process by Using Fuzzy Logic Based Technology. International Conference on Information and Software Technologies 2016 Oct 13 (pp. 119-131). Springer International Publishing. https://doi.org/10.1007/978-3319-46254-7_10.

[7] Guler H, Kilic U, Kaya T (2016), The Design of Fuzzy Based Weaning Protocol in LabVIEW Environment. Proc. International Journal of Artificial Intelligence and Neural Networks. 6: 16-20.

[8] Gates RS, Chao K, Sigrimis N (2001). Identifying design parameters for fuzzy control of staged ventilation control systems. Computers and Electronics in Agriculture. 31(1):61-74. https://doi.org/10.1016/S0168-1699(00)00174-5.

[9] Villafáfila-Robles, R., \& Salom, J (2012), Heat, Ventilation and Air Conditioning (HVAC). Electrical Energy Efficiency Technologies and Applications. 1:

335-355. https://doi.org/10.1002/9781119990048.ch11.

[10] Villafáfila-Robles R, Salom J. (2012), Heat, ventilation, and air conditioning (HVAC), A. Sumper, A. Baggini (Eds.), Electrical Energy Efficiency: Technologies and Applications, John Wiley and Sons, Chichester, UK, pp. 335-356. https://doi.org/10.1002/9781119990048.ch11.

[11] Zhang L, Liu X, Jiang Y (2013). Application of entransy in the analysis of HVAC systems in buildings. Energy. 53:332-42. https://doi.org/10.1016/j.energy.2013.02.015.

[12] Veleva V, Hart M, Greiner T, Crumbley C (2013), Indicators for measuring environmental sustainability: A case study of the pharmaceutical industry. Benchmarking an International Journal. 10(2):107-19.

[13] Schneider JL, Wilson A, Rosenbeck JM (2010). Pharmaceutical companies and sustainability: an analysis of corporate reporting. Benchmarking: An International Journal.17 (3):421-34.

[14] Avgelis A, Papadopoulos AM (2009). Application of multicriteria analysis in designing HVAC systems. Energy and Buildings. 41(7):774-80. https://doi.org/10.1016/j.enbuild.2009.02.011.

[15] Ahamed NU, Taha ZB, Khairuddin IB, Rabbi MF, Rahaman SM, Sundaraj K (2012). Fuzzy logic controller design for intelligent airconditioning system. In Control Science and Systems Engineering (ICCSSE), 2016 2nd International Conference on 2016 Jul 27 (pp. 232-236). IEEE

[16] Ahamed NU, Yusof Z, Hamedon Z, Rabbi MF, Sikandar T, Palaniappan R, Ali MA, Rahman SM, Sundaraj K (2016). Fuzzy logic controller design for intelligent drilling system. In Automatic Control and Intelligent Systems (I2CACIS), IEEE International Conference on 2016 Oct 22 (pp. 208-213). IEEE. https://doi.org/10.1109/I2CACIS.2016.7885316.

[17] Xu M, Li S, Cai W (2005), Practical receding-horizon optimization control of the air handling unit in HVAC systems. Industrial \& engineering chemistry research.44 (8):2848-55. https://doi.org/10.1021/ie0499411.

[18] Jimenez L (2011), Molecular applications to pharmaceutical processes and cleanroom environments. PDA Journal of Pharmaceutical Science and Technology. 65(3):242-53. https://doi.org/10.5731/pdajpst.2011.00730.

[19] Whyte W, Eaton T. A cleanroom contamination control system. European Journal of Parenteral and Pharmaceutical Sciences. 7(2):55-61.

[20] Whyte W, Eaton T (2004), Microbial risk assessment in pharmaceutical cleanrooms. European Journal of Parenteral and Pharmaceutical Sciences. 9(1):16-23.

[21] Ahamed, N. U., Benson, L., Clermont, C., Osis, S. T., \& Ferber, R. (2017). Fuzzy Inference System-based Recognition of Slow, Medium and Fast Running Conditions using a Triaxial Accelerometer. Procedia Computer Science, 114, 401-407. https://doi.org/10.1016/j.procs.2017.09.054.

[22] Ahamed, N. U., Taha, Z. B., Khairuddin, I. B. M., Rabbi, M. F., Sikandar, T., Palaniappan, R., Sundaraj, K. (2016, October). Development of fuzzy inference system for automatic tea making, IEEE International Conference on Automatic Control and Intelligent Systems (I2CACIS) (pp. 196-201). 